\title{
Health Visitors' Work in a Multi-ethnic Society: a Qualitative Study of Social Exclusion
}

\author{
A. M. BOWES* and T. MEEHAN DOMOKOS $†$ \\ (Received 19.6.97; Accepted 5.9.97)
}

\section{ABSTRACT}

Health visiting is adopting an enabling model of practice, which may promote social inclusion, but is under pressure to justify itself. The article focuses on health visitors' work with Pakistani women and comparable white women in Glasgow, examining the nature of health visiting and women's responses to it. Health visitors' perspectives involve the appreciation of cultural differences, building relationships with clients, and some stereotyping of clients. Techniques include highly valued home visiting, and processes of negotiation with clients. Problems faced include difficulties with interpreters, lack of training, relationships with other professions, recent changes in the NHS, and issues of stress and personal safety. Women's views of health visitors are generally positive, especially concerning home visits, time spent with clients, and gate-keeping access to GPs. Negative views came mostly from white women, and concerned the more controlling models of health visiting. Thus, enabling health visiting practice was widely appreciated, and could act as an inclusionary force, facilitating access to and use of health services. Exclusion was operating at institutional level, towards minorities and women of lower socio-economic groups, but was being actively resisted by practitioners.

\section{INTRODUCTION}

As representatives of a universal service, health visitors in Britain have unique access to the lives and homes of all citizens, and one of their major roles is working with mothers and their children under five years old. There is now a developing research literature on the nature of health visiting (e.g. Chalmers, 1992, 1993, 1994; Cowley, 1995a; Delacuesta, 1993), which emphasises a change in health visiting practice from a

\footnotetext{
* Reader in Sociology, Department of Applied Social Science, University of Stirling, FK9 4LA, Scotland.

$\uparrow$ Formerly Research Fellow, Department of Applied Social Science, University of Stirling
} 
formerly more directive approach (Symonds, 1991) to an enabling role, involving advocacy and empowerment of clients, especially those in disadvantaged circumstances, excluded in various ways from access to effective health care and support. Rolls (1992) is especially supportive of this shift in practice, arguing that it helps to minimise potential power differences between health visitors and their clients, and thus to facilitate health promotion and health support. Mayall (1990a, p. 220) similarly argues that the 'policing' elements in the health visitor-client relationship can be detrimental to effective health promotion work. She adds that some minority mothers, especially those newly arrived in Britain, looked to their health visitors as a major source of support in their childcare efforts. These writers suggest that health visitors' more recent work may have a key role to play in alleviating disadvantages in health, such as those identified by Oakley, Popay and Watson (1996) as experienced by minority ethnic groups, lower socio-economic groups and women.

Whilst research evidence is thus presenting health visiting and its potential in a generally positive light, the profession is under considerable pressure to justify and quantify its work (Carney et al., 1996; Cowley, 1995a; MacDonald et al., 1997). The profession has been much reduced in size in recent times, and protocols now restrict the amount of universal home visiting, seen as central to the enabling practice identified above. Health visitors are increasingly required to record their tasks, and identify their outcomes (Carney et al., 1996). Health visiting remains difficult to evaluate: for example, Barker (1992) argues that of its nature, it cannot be evaluated using conventional evaluation tools, especially quantitative indices, and that an 'action research approach', which adopts more qualitative methods is necessary. Cowley (1995b) sees health visiting work as centrally uncertain and ambiguous. Delacuesta (1993) adds that the boundaries of health visiting are not clear, and that 'fringe work', which does not fit management protocols, is constantly generated and can be eventually incorporated into protocols. Her work suggests that health visitors themselves can actively influence the developing nature of their profession (and cf. Dingwall, 1976). Appleton (1996) however, cautions that health service managers do not necessarily recognise the importance of health visitors' work.

Thus, on the one hand, health visiting appears a potential force against social exclusion of disadvantaged groups, and, on the other, it seems to be under threat, from a service which aims at 'health for all' (WHO, discussed by Ahmad, 1993). There is a clear need for research 
which focuses on health visiting at the point where it may challenge social exclusion, and examines the consequences of current policy trends. It may be that the pressure on health visiting promotes social exclusion, as enabling services are progressively denied to the most disadvantaged social groups.

In this article, we examine health visiting work in relation to a sample of Pakistani ${ }^{1}$ mothers in Glasgow, and a comparator sample of white mothers, living in the same areas of the city. We consider how the health visitors were working with both groups of women, the women's responses to this work, and the ways in which social inclusion was being promoted. We also discuss the effects of recent policy changes, and argue that these indeed risk reinforcing processes of exclusion.

THE RESEARCH STUDY

The data on which our argument is based were collected in 1994-5 as part of a larger study of communication between Pakistani women, white women, health visitors and general practitioners (Bowes and Domokos, 1996, 1997). ${ }^{2}$ The data consist of semi-structured interviews with 62 women of Pakistani heritage, who were mothers of one or more children under five, 68 white women living in similar areas, 50 health visitors and 25 general practitioners. ${ }^{3}$ All the samples were random, and the samples of women were weighted to ensure representation of a range of socio-economic groups, using housing area type as a proxy.

All but one of the health visitors interviewed were women, and two had expert knowledge of Punjabi, the local language of many Pakistani Glaswegians. Their median years of experience was 13 (range 1-26) and their caseloads of Pakistani mothers varied: 29 had fewer than ten, nine had fifty or more (227 was the highest). Several had formerly worked with Pakistani clients, but were not currently doing so.

The median age of the Pakistani women was 30 and of the white women 33. Pakistani women had a median of three children, and white women, two. Pakistani women were more likely to live in an extended family ( 27 per cent, as compared with 4 per cent), less likely to have a job outside the home ( 5 per cent, as compared with 38 per cent) and somewhat more likely to have relatives living nearby (85 per cent as compared with 73 per cent). White women's housing circumstances showed a bigger range, being both better, and worse, than Pakistani women's. Pakistani households tended to be larger, and there was more pressure on living space.

The interviews with women covered their contacts with and views 
about health visitors and general practitioners, especially as related to their role as mothers. Interviews with health visitors covered their experiences of work with Pakistani and white women, their training in the area, their evaluation of services, and cooperation with other professionals, especially general practitioners.

All the interviews were transcribed, and then indexed, using categories derived from the interviews themselves, thus preserving the views of respondents, and avoiding the imposition on the data of preconceived categories. The scrutiny of data was then completed with the aid of the NUD.IST software. In presenting the data, we refer to patterns which have been established through systematic analysis, and include representative quotations. ${ }^{4}$ Data analysis for this article focused on the questions of health visitors' approach to their work with minority ethnic women, whose language and culture they do not generally share, health visitors' views of the service they offer, and the views of Pakistani women and white women on the health visiting service.

\section{HEALTH VISITING}

\section{Health visitors' views of their work}

The health visitors had a variety of perspectives on work with Pakistani families. There was a strong tendency to see differences between Pakistani women and white women in terms of culture, especially language and family relationships, family life and routines, though the differences were often felt to be less significant for those women of Pakistani heritage born in Glasgow. In terms of women's needs, differences were seen as less clear, in that, for example, all women might become depressed and need health visitor support, even though the particular triggers of their depression might differ. So a white woman might lack support without nearby relatives, whereas a Pakistani woman might lack support within a group of relatives. Similarly, all new mothers might need advice about infant feeding, whilst experiencing different particular problems: for white women, the use of sugary juice drinks was a frequently mentioned issue, whereas for Pakistani women, prolonged use of formula milk was seen as a problem. There was some awareness of the potential hostility of the local environment for Pakistani women, though this was not widespread:

If these Pakistani girls are in the house on their own, they have no transport, don't know anyone else. I found tremendous loneliness - very afraid to go out to other girls. I think perhaps they thought they might be seen as being different, and they find great difficulty in relating to other girls in the neighbourhood. (HV309) 
HV321 also spoke about the greater isolation of young Pakistani mothers, who would not necessarily be welcomed by local mother and toddler groups. Mayall's (1990a, p. 212) study also suggested that this was the case.

Health visitors who had extensive experience of working with Pakistani families spoke at length about cultural differences between themselves and their Pakistani clients. They generally did so in a sensitive and subtle way, and there were few examples of crude stereotyping which other commentators (e.g., Bowler, 1993; Stubbs, 1993) have seen as central to the relationship between minority ethnic women and health professionals. These experienced health visitors gave detailed accounts of how they had come to work with Pakistani women with little training or other preparation, and spoke about making mistakes and learning from them some distinctive strategies which they would then use when working with Pakistani women. For example, several spoke about relationships within families, and the need to show proper respect for these, including the role of the mother-in-law, and the value which women clients attached to their family relationships. They were also conscious of the need not to stereotype people, and the maintenance of a focus on the individual woman was a strong theme: this seems to reflect the recent shift towards enabling and supporting relationships with clients (Chalmers, 1992, 1993; Cowley 1995a):

Part of our health visiting principles is that we are non-judgemental. (HV310)

They're all different. They all have different moods, and I think you've really got to adjust yourself to that family's needs, so you're basically assessing a situation as you go in. (HV318)

There were many parallels described between building relationships with Pakistani and white women, involving discussion of the need to approach people step by step, to build good relationships with them, and to use these as the basis for health promotion work. Some of the problems were seen as similar between the two groups of women. For example, although language was generally seen as a much bigger issue for Pakistani women, health visitors also spoke of linguistically based communication difficulties with white women. Also, the role of grandmothers was discussed for both groups of women, especially the tendency of grandmothers to take over care of the child in threegeneration households. The drawing of parallels in this way indicates that health visitors were resisting the consistent construction of Pakistani women as 'other'. HV322 spoke of the need to avoid attributing all Pakistani women's problems to their ethnicity: 'I think 
there's not as many differences as people maybe think ... You could say "That's just because you're Asian, and that's your problem"... [but] I don't think so' (HV322). She went on to discuss the effects of overcrowded living conditions on women's experiences, and on the problems they might face. Similarly, HV328 preferred to emphasise similarities rather than differences: 'Most of my Pakistani families are just like everybody else in my practice' (HV328). She felt that there was a tendency to concentrate on the 'dramatic and sensationalist' problems experienced by Pakistani women, and that cultural differences could be emphasised disproportionately.

Health visitors who had less experience of work with Pakistani women were more likely to speak negatively about them. One strong theme was the issue of nursery places, whereby Pakistani families were said to be claiming priority places unjustifiably because English was a second language. This seems to have been an issue locally at the time of the interviews (i.e., not a general issue), but illustrates how negative characterisations of a group seen as distinct could come into play. The negative view was influencing practice, in that applications from local Pakistani families were being scrutinised especially carefully. Some health visitors felt that Pakistani women were inclined to be especially demanding of services: '[They] can be quite demanding, can be quite manipulative, and can make the system work to their advantage, much more so than some of the indigenous population' (HV310). Here, there are parallels with the work of, for example, Bowler (1993) and Torkington (1995), and others, who observe exclusionary stereotyping in practice. But in the present study, these were minority views. Moreover, exclusionary typifications were also in use for other categories of client: some white families were said to have distinctive characteristics that were never mentioned for Pakistani families: 'Usually, it's due to drug addiction, and that takes priority. And they're in and out of prison, and [have] various problems' (HV319).

\section{Techniques of health visiting}

Throughout the interviews with health visitors, there was a strong emphasis on the value of home visits: these were felt to be under threat due to new protocols, management priorities, and GP contracts. The health visitors interviewed were here in agreement with trends discussed in the research literature by, for example, Butler 1997, Campbell and MacDonald (1995), MacDonald et al. (1997), Reynolds 1996 and Wiles and Robison (1994). The home visits were seen by nearly all the health visitors interviewed as central to their work, and they spoke 
about the value of meeting people on their home ground, of understanding more about them thereby, of seeing them in their ordinary surroundings, and seeing faces other than were 'put on' at clinics. Home visits were seen as fairer for clients:

If a girl is very upset, and can be emotional, and the tears come, it's very distressing for her, if she's sitting in a body of people [at a clinic] and weeping and wailing. And if she feels that is likely to happen, she would rather I go to the house, and I would rather go to the house. (HV309)

Health visitors argued that building relationships with their clients was central to their work, especially their preventative, trouble shooting and supporting work with all women. HV309, for example, argued that her long-term knowledge of families enabled her to raise and deal with problems that might otherwise remain hidden. HV330 spoke of the value of investment: 'Because I know my families very, very well, any change in that person...you pick up on it' (HV330). Similarly, HV323 discussed the value of her knowledge: 'I really feel......we know a lot about the patients that probably GPs don't know about' (HV323). She added that the GPs did not really recognise the value of this knowledge for their own practice. HV340, discussing the identification of postnatal depression ${ }^{5}$ explained how she used her knowledge of families, and the home visit:

You just get this feeling in the house when there is something not quite right, and it is a very difficult thing to put your finger on. So you tend to go back, and go back...until you think 'Oh, that's what it is. Right.' (HV340)

There are echoes in statements like this of Reynolds' (1996, p. 103) findings concerning health visitors' use of 'gut feelings' and 'extrasensory perception' in their practice. HV344 explained how she saw this aspect of her work: 'I think we are in a very privileged position, and people do tell you things. They will tell you things that they would never tell anyone else. I am constantly amazed by that' (HV344).

In many respects, home visiting was seen as more important for Pakistani women. Where there were language differences, home visits allowed time for women to talk more fully, and for health visitors to understand them. Where health visitors were unfamiliar with Pakistani cultures, they had found home visits an important way of learning to appreciate cultural differences, and to learn appropriate ways of acting.

As a service which relies on the goodwill of clients to gain entry to their lives, and on building good relationships in order to influence client behaviour, health visiting is inevitably involved in processes of negotia- 
tion with clients, as Chalmers (1992), Delacuesta (1993) and others have noted. There is thus considerable pressure on health visitors to develop sensitivity to their clients, and to approach their work reflexively, and in ways which respond to individuals. Throughout the interviews with health visitors, it was clear that this was generally their own view of the approach they tried to take in their work with all client groups, including Pakistani women. In forming relationships with clients, they described how they used aspects of their own experience to lay out meeting grounds between themselves and their clients. For example, several referred to the experience of motherhood, which enabled them to express empathy with mothers who were worrying about or having difficulties with their children. Specifically in relation to working with Pakistani women, others discussed experiences of being an outsider, or of a particularly strict upbringing, which they felt had helped them in their work.

Some of the health visitors reflected on their relationships with Pakistani women in a more general way. They referred to their own outsider status in relation to the Pakistani community, and discussed how this could be a source of difficulty in working with Pakistani women, as we have noted. But they also suggested that their outsider status could be an asset, and that as 'professional friends' they were seen as maintaining confidentiality, and helping women deal with issues which were difficult or impossible to raise in the family: all women might face such issues, but the health visitors felt that for Pakistani women, some, such as leaving home, were particularly significant.

The nature of modern health visiting as an enabling profession was emphasised explicitly by several health visitors: 'I see myself as not making decisions for people, but to help them come to a decision, and support that decision. Or to get them to be their own advocate' (HV331). Referring to the old style of health visiting, which she felt had been more directive, HV333 said, 'I think that was wrong, because you are taking people's authority to make decisions away from them' (HV333). Here, the evolution of health visiting practice away from the older, more authoritarian style (Welshman 1996) can be observed directly.

\section{Problems in health visiting}

Work with Pakistani women was seen as more difficult, but often more rewarding, than work with white women, but health visitors discussed many problems with such work. The problems discussed here are the use of interpreters, lack of training, issues of interprofessional co-operation and division of labour, workload, and personal safety. Most of these 
were encountered by the health visitors in other areas of their work, but were of particular importance in their work with Pakistani women.

There were many accounts of problems using interpreters. As noted, few of the health visitors had language skills, and interpreters were needed in work with Pakistani women who did not speak English. Health visitors were very concerned about using family members as interpreters, usually for reasons of confidentiality, and sometimes because family members were seen as speaking for women. Professional interpreters were also seen as problematic: access to them was difficult, translations were often only partial, sometimes translations were "censored', interpreters were not necessarily aware of medical terminology in either language, and the issue of confidentiality remained. Some health visitors spoke more positively about the use of health trained advocates, rather than interpreters. The characteristics of the interpreters themselves were also discussed. For example, HV312 discussed the need for the interpreter to share religion with the client, then added issues of gender and age:

If it's an older woman, or a menopausal woman, I wouldn't use someone that was very young, because they would be embarrassed talking ... you really need to try and get someone that would be a wee bit older [so that] they've got more empathy with her. (HV312)

Lack of training was seen as a problem, especially for the older health visitors: those more recently trained had received lectures from health visitors experienced in working with Pakistani families, and felt that this had alerted them to the issues they were facing in their work. Some health visitors had sought in-service training, frequently in their own time and at their own expense: one in particular had asked for language training, but this had been refused. Lack of training, health visitors felt, meant that they lacked knowledge of Pakistani cultures, and had to learn on the job: whilst they saw making mistakes as a valuable learning experience, they did not see practising on clients as the best way of acquiring knowledge. For example, HV312 described having made mistakes, discussing a child in an extended family with a woman who was not the mother, and explained the need to check whose child was whose: 'That's something I learned on the floor, which I felt that if I'd known that before, it would have helped quite a bit' (HV312).

Health visitors often spoke of their skill at identifying problems and of the need to refer their clients to other professionals for the necessary assistance. Whilst several felt that their relationships with the GPs with 
whom they worked were good and that communication with GPs was effective, there were many who saw problems in this area: some complained that GPs referred cases to them inappropriately, expecting them to do work outwith their remit, including the work of the GP. A strong theme in the interviews was the issue of relationships between social workers and health visitors. Welshman's (1996) account suggests that such relationships have long been somewhat difficult. Where the health visitors discussed them, they were invariably described as problematic, with complaints about duplication of effort, professional territorial disputes, and lack of responsiveness from social workers. These problems appeared at least partly related to the changes in health visitors' roles towards routinisation, as well as the development of community care policy (in which professional divisions of labour appeared unclear), and some aspects of changes in structure in the NHS, including GP fundholding, which led GPs to prioritise certain activities, and to expect health visitors to concentrate on them. Some were apparently attempting to use health visitors as practice nurses and as district nurses, as well as health visitors. These problems associated with NHS changes have been widely discussed, for example by Campbell and MacDonald (1995), who see the increased emphasis on clinic-based work as bringing about shifts in the nature of health visiting work, and Gibbings (1995), whose discussion of skill mix suggests that health visiting work is in danger of being marginalised. The health visitors interviewed felt that this set of problems was also reducing their ability to offer high quality services and that clients who needed home visits, time and advice were therefore losing out: again, those who lost out the most were the most deprived in socio-economic terms, both white and Pakistani, and those whose access to the service was already restricted by linguistic and cultural factors, particularly Pakistani women.

Workloads were, health visitors argued, increasing, both in terms of numbers of clients and also in terms of the number of tasks required. Many of these tasks involved paperwork, which, though seen as probably necessary, was resented as very time consuming: many felt that clerical assistance with the paperwork would allow more time to be spent with clients. Some respondents discussed the difficulty of making their work fit into the quantitative record keeping required by their managers: Contrasting her skill with that required to tick boxes on forms, HV320 argued 'A trained monkey could do this [tick boxes]' (HV320). She felt her skill was undervalued by managers. Again, these concerns are reflected in wider discussions (e.g., Cowley, 1995a; 
Gibbings, 1995; MacDonald et al., 1997). There was a strong feeling that health visiting was under threat, at least partly because it was difficult to quantify: 'It's a luxury they're not going to afford' (HV311). The work overload in deprived areas was seen as especially serious: managers were apparently concerned with numbers of clients, rather than the nature or seriousness of their needs. Several health visitors described having moved jobs to get away from the particularly stressful conditions of work in parts of the city: 'My way to cope with it eventually was to get out of it' (HV350).

A further source of stress was the issue of personal safety: health visitors carried personal alarms, they followed careful safety strategies in the street and in clients' houses and many described fearing for their safety. Certainly, they felt that these stresses and fears were detrimental to their work, and to their ability to provide an effective service to all their clients. Again, it was clear that the most needy, who lived in the most dangerous areas and needed more time from their health visitors, were losing the most. For example, HV314 spoke of homeless women who were being rehoused on the fourteenth floor of tower blocks, and the fear she felt visiting them:

You have to go up in the lift, and I find it quite frightening because it stops, and it seems to be always deserted as well. You get in the lift, and it opens at this floor, and you don't know who is going to get in ... You have quite a lot of girls [there], and you have to go. But I do find it quite stressful. I feel very vulnerable. (HV314)

Clearly, the women she was visiting were also very vulnerable.

Resources for health visiting

From the health visitors' point of view, the service they provided was vital to the effective provision of health services generally. Their health promotion work was preventative, their interpersonal skills with clients were important to its effectiveness, they could act as facilitators for clients seeking health care, encourage the appropriate use of services, as well as dealing with issues of child health surveillance. We have argued, however, that much of this work has been called into question, following recent NHS reforms (Butler, 1997; Gibbings 1995).

Among the health visitors interviewed, there was a significant fund of experience of and knowledge about working with Pakistani women in Glasgow. There was evidence that this was being shared between health visitors, with the less experienced seeking specialist advice from those who were known to be expert: they had also been used as training resources. Their knowledge was greater than that of most of the 
GPs interviewed, and appeared greater than that of other professionals. This is suggested by some other research (Bowes and Dar, 1996), which found poor knowledge and little experience of work with Pakistani families among local social workers. In particular, health visitors seemed to be getting support in their work from other health visitors. Many spoke of the value of their meetings with one another in these terms. They did not seem to be receiving institutional support (cf. Holden, 1996; Willard, 1996) in their day-to-day work, in terms of training (cf. Nolan, Owens and Nolan, 1995), and in terms of resources, such as access to interpreters.

WOMEN'S VIEWS OF THE HEALTH VISITING SERVICE

We have argued elsewhere (Bowes and Domokos, 1996) that the research findings indicated that women felt communication was better with health visitors than it was with GPs. In scrutinising the data on responses to health visiting, we attempted to identify what it was about health visiting that led women to this view, and looked in more detail at what women felt they got from their contacts with health visitors. The more effective communication with health visitors was recognised by the women: 'You can talk more freely to the health visitor than the doctor' (PW146). 'You want to talk to someone, but you can't talk to your family. She's the one that I talk to, and she's very helpful' (PW147). PW147 was experiencing serious marital problems and had received considerable support from her health visitor. White women were also generally positive about their health visitors in terms of communication: 'I had a lot of faith in her ... trusted everything she said' (WW237).

The home visits were generally much appreciated, and women described how they felt the health visitors were taking time to listen to them, and understand their problems.

The main thing is that they listen to you. (PW133)

I [liked] it if she came to the house, because then I can ask her a lot of questions, as much as I want. (PW135)

TMD: What makes it easier to tell her? PW147: The way she talks to you. She's always smiling, and very, very softly, she tells you everything ... The good thing [is], she always listens to you first. My English is not very good, right...but she always, she understands me. (PW147)

This woman explained that she could manage to speak in English, but not if she was rushed, and that, therefore, the home visits were a particular advantage. A white woman explained why she found clinics 
difficult: 'There's no privacy at all, which I don't think leads to a good relationship' (WW238).

The appreciation of home visits was especially marked for Pakistani women (as in Mayall's 1990b study of a multi-ethnic sample of women), many of whom commented positively on the health visitors' attempts to understand their particular preferences: like the health visitors, they felt these were different from those of white women. 'I think they know quite a lot about our culture' (PW107). PW107 explained the importance of this knowledge, and incidents in which the health visitor had been able to override her mother-in-law's views about childcare, which she was finding difficult to resist. She explained:

She's more important than the doctor, especially when you've got a baby...we need someone really, really helpful, really understanding...You are relying on her. The health visitor is quite important, and they ... should sympathise with you. (PW107)

Both white and Pakistani women described health visitors who had helped them use GP services more effectively, either by reassuring them that they did not need to see GPs, or by supporting them in their efforts to be seen and 'taken seriously'. For example, PW106 explained how the health visitor could help: 'There are things you could maybe talk over, you know, give you peace of mind...instead of just going up to the surgery' (PW106). PW108 described how the health visitor had telephoned the GP for her on many occasions. PW143 had been accompanied on her visits to the GP by her health visitor, who had helped her communicate her health problems to the GP. PW120 had had problems convincing her GP that her child was ill, and had been told by the health visitor 'Take him back again. Keep on taking him until the doctor listens to you' (PW120).

WW209 had found it difficult to approach her GP, but had been encouraged by her health visitor: "She would say "You know I think you should go in and see the doctor. I think you should do it." I think when you've just had a baby, you're very vulnerable' (WW209). She had especially valued the health visitor's support at that time. WW225 had suffered from post-natal depression and related how her health visitor had helped explain her condition to her, and had acted as an advocate for her with her GP, who had only offered leaflets, which WW225 could not understand, as explanations. These findings generally support research on health visitors which has promoted their potential as advocates (e.g., Campbell and MacDonald, 1995; Willard 1996).

For some women, the health visitor provided much valued general support: PW101, for example, described how she had felt very alone 
when first married, separated from her natal family, and how the health visitor had been an important source of support. 'I know what it's like ... and when I think about someone who starts new, who gets married and has children, or starts a new life, and its very difficult for them having no-one from outside to contact' (PW101). This case compares with Mayall's (1990b) findings regarding women's needs for social support. Other women described having relied on their health visitors in times of need. WW114, whose baby was particularly demanding, explained 'She [health visitor] saved my life, she really did...This was a problem child, and she was always there for me' (WW114).

Nearly all the negative comment on health visitors came from white women, who referred to health visitors who had acted, as they saw it, autocratically or threateningly, leading to worries that their children might be removed. One went as far as to say 'Health visiting is a load of rubbish' (WW229). But these women who were negative had generally managed to change health visitors, and took a more positive view of their current one. Some white women were somewhat nervous of health visitors, seeing them as watching them: for example, WW231 felt that too frequent visits would be unnerving ' $I$ 'd feel there was something wrong, that I'd been watched or something' (WW231).

Another woman did not like the health visitor asking questions which she felt had nothing to do with her child: 'She was asking questions while we were there to get the wean ${ }^{6}$ checked oot. She was asking all these questions that were totally irrelevant' (WW233). She had not appreciated the health visitor's attempt to get to know the wider circumstances of her child's life, and felt the questions were intrusive. Another woman felt that the health visitor's main purpose was not to help her, but to instruct her: 'She was...nice enough, but I felt she was telling you what to do...she wasnae giving you advice, or suggesting things, it was like "We know better', and...that was the impression I got of her" (WW239). This woman had managed to move on to another health visitor, who she described as 'Really, really great' (WW239). She had noticed a change in health visiting practice over the seven years between her two children, towards a more flexible approach. WW240 spoke of one health visitor who, she felt, undermined mothers' confidence: 'She's really not interested in what she's doing...people avoid her like the plague ... They're all terrified of her' (WW240).

WW241 explained that she preferred less contact with the health visitor, once she had a second child:

I desperately needed my first one [health visitor] for my first, but by the time [my daughter] was born, I didn't feel the need, and really, I found it more of an intrusion ... if 
she came in too often, because I was doing my own thing, and I was getting on with it. (WW241)

These white women were identifying health visiting as a more controlling and policing profession, instructing incompetent women in the skills of childcare (as described by Symonds, 1991 and Welshman, 1996). They clearly preferred the more enabling approach described by the health visitors interviewed.

Pakistani women who had had bad experiences with health visitors were rather few. One (PW144) complained that the health visitor kept referring her to a social worker, despite her repeated attempts to explain that she could not get on with the particular social worker who came. Others felt that the health visitor had not come to see them often enough, but commonly added that this was not really a problem, as, with their second child, they did not need as much advice.

Certainly, one factor in the relative success of health visitors' relationships with their clients was gender: shared gender was seen as a factor promoting good relationships for both white and Pakistani women, and health visitors known to have children themselves were seen positively. This echoes the health visitors' own comments on the value of their own experience for helping build relationships with their clients.

\section{CONCLUSION}

From the health visitors' point of view, there was a clear rationale underlying their work, of sensitivity to clients, of proceeding step by step, of adopting an enabling, rather than a directive role. Here, they appeared consciously to be moving away from the more controlling models of health visiting identified in some literature (e.g., Mayall, 1990b; Symonds, 1991; Welshman, 1996). When they described problems with their work, they pin-pointed threats to their ability to pursue an enabling role, including workload in the form of paperwork in particular, caseload, in terms of numbers of clients, and the pressure to do work which was quantifiable, and easily definable, which fitted what they saw as management models. They were especially concerned about the threat to home visiting, which they saw as central to their practice with all women, and especially Pakistani women. In their eyes, the managerial pressures to routinise their work were operating against their attempts to implement enabling practice. In their experience, the management-driven direction of change was operating to exclude those who most needed the enabling health visiting practice. Further restrictions on their work came from lack of training and lack of access to appropriate interpreters. 
Linked with these problems were issues attached to the potential for health visitors to act as gateways to other professionals, notably GPs and social workers. GPs relationships with health visitors appeared to be changing, as fund-holding practitioners tried to use health visitors differently, and to alter their role. Health visitors' complaints about their relationships with social workers appeared related to professional divisions of labour in community care: health visitors experienced these as deskilling, and devaluing their work. Furthermore, health visitors faced stress in their work in more deprived areas of the city, and there was clear potential for this to drive experienced and skilled practitioners away from their work. Thus, health visitors' preferred model of work was under threat, and the health visitors themselves reflected concerns expressed in recent research literature (such as Cowley, 1995a; Gibbings, 1995; Butler, 1997).

Women who valued health visiting valued exactly those elements of it which the management-led changes appeared to be threatening, and which the health visitors themselves wanted to preserve and develop. Women valued the time health visitors spent with them, their thorough knowledge of women and their circumstances, their ability to listen and understand women's concerns and wishes. Pakistani women appeared to value these qualities more strongly. Where health visiting was criticised, unpopular aspects of practice appeared to be those which were more directive, and which were interpreted as interference or bossiness. It appeared therefore that the women generally appreciated the more enabling health visiting practice. These findings contrast somewhat with those of Mayall (1990b), who found greater differences of view between health visitors and their clients.

Our research suggests that enabling practice could operate as an inclusionary force, facilitating the access of potentially excluded clients to health services, and promoting their effective use. However, the aspects of health visiting which appeared to promote inclusion were under threat. Thus, in contrast to other research on the role and behaviour of health professionals, which has identified racism built into their actions, our work has emphasised exclusion operating at an institutional level, and has indicated that this group of professionals actively resist it. The exclusionary forces are, moreover, wider than institutional racism, in that the practices most valued by Pakistani women are also especially valued by white women, and much needed by those in lower socio-economic groups. Changes in regime thus threaten or remove these practices for all such women, to the detriment of their access to effective health services. 
The clearest implication for health services offered by this work is a need to reevaluate those recent changes which apparently have exclusionary effects. The potential value of enabling health visiting practice for promoting good health and access to health services for deprived groups can be seen as an asset. With so much evidence of racism operating in health services, and racism seemingly so firmly entrenched (see e.g., Stubbs, 1993; Esmail and Everington, 1997), health workers who promote inclusion offer some hope of improvement.

\section{NOTES}

1 The term 'Pakistani' is used to denote the ethnic background of one of the groups of women interviewed, and is a term they used themselves. The term 'South Asian' is used to denote people with origins in the Indian subcontinent as a whole. Other terms, such as 'Asian', appear where respondents or literature sources have used them. It should be noted that the terms 'Pakistani' and 'white' (used for the other group of women) do not imply ethnic uniformity within the categories.

2 The research was funded by the Chief Scientist Office, Health Department, the Scottish Office.

3 The data from GPs are not discussed in this article.

4 All quotations are attributed. PW denotes a Pakistani woman, WW a white woman, and $\mathrm{HV}$, a health visitor.

5 The literature suggests that health visitors have mixed success in their efforts to identify depression (Holden 1996; Sheppard, 1996).

6 'Wean' = child.

\section{REFERENCES}

W. I. U. Ahmad (1993), 'Promoting equitable health and health care: a case for action', in W. I. U. Ahmad (ed.), 'Race' and Health in Contemporary Britain, Open University Press, Buckingham.

J. V. Appleton (1996), 'Working with vulnerable families - a health visiting perspective' Journal of Advanced Nursing 23: 912-18.

W. Barker (1992), 'Health visiting: action research in a controlled environment' International Journal of Nursing Studies 29: 251-9.

A. M. Bowes and N. S. Dar (1996), Pathways to Welfare for Pakistani Elderly People in Glasgow, Scottish Office Central Research Unit, Edinburgh.

A. M. Bowes and T. M. Domokos (1996), Issues of Communication between General Practitioners, Health Visitors and Women of Pakistani Heritage, Final Report to Chief Scientist Office, Scottish Office Department of Health, Edinburgh

A. M. Bowes and T. M. Domokos (1997), 'Pakistani women, general practitioners and health visitors: communication and service access', in A. M. Bowes and D. F. Sim (ed.), Perspectives on Welfare: the Experiences of Minority Ethnic Groups in Scotland, Ashgate, Aldershot.

I. Bowler (1993), 'They're not the same as us: midwives' stereotypes of South Asian descent maternity patients', Sociology of Health and Illness, 15: 157-77.

J. R. Butler (1997), 'Child health surveillance in England and Wales: the bad news', Child: Care, Health and Development, 23: 339-54.

H. Campbell and S. MacDonald (1995), 'Evaluation of the Fife health visitor pro-active training program', Public Health, 109: 327-35.

O. Carney, J. McIntosh, A. Worth and J. Lugton (1996), Assessment of Need for Health Visiting, Department of Nursing and Community Health, Glasgow Caledonian University, Glasgow.

K. I. Chalmers (1992), 'Giving and receiving: an empirically derived theory on health visiting practice', Journal of Advanced Nursing, 17: 1317-25. 
K. I. Chalmers (1993), 'Searching for health needs: the work of health visiting' Journal of Advanced Nursing 18: 900-11

K. I. Chalmers (1994), 'Difficult work: health visitors' work with clients in the community', International Jounral of Nursing Studies, 31: 168-82.

S. Cowley (1995a), 'In health visiting, a routine visit is one that has passed', Journal of Advanced Nursing 22: 276-84.

S. Cowley (1995b), 'Health-as-process: a health visiting perspective' Journal of Advanced Nursing 22: 433-41.

C. Delacuesta (1993), 'Fringe work: peripheral work in health visiting', Sociology of Health and Illness, 15: 665-82.

R. Dingwall (1976), 'Accomplishing profession', Sociological Review, 24: 331-49.

A. Esmail and S. Everington (1997), 'Asian doctors are still being discriminated against', British Medical Journal, 7094, 31 May. (http://www.bmj.com/bmj/archive/70941.htm)

S. M. Gibbings (1995), 'Dependency, skill mix and grade mix and their effects on health visiting practice', Journal of Clinical Nursing, 4: 43-7.

J. Holden (1996), 'The role of health visitors in postnatal depression', International Review of Psychiatry, 8,79-86.

A. L. MacDonald, I. H. Langford and N. Boldero (1997), 'The future of community nursing in the United Kingdom: district nursing, health visiting and school nursing', Journal of Advanced Nursing, 26: 257-65

B. Mayall (1990a), 'A joy or a hassle: child health care in a multi-ethnic society', Children and Society, 4: 197-224.

B. Mayall (1990b), 'The division of labour in early child care - mothers and others', Journal of Social Policy, 19: 299-330.

M. Nolan, R. G. Owens and J. Nolan (1995), 'Continuing professional education: identifying the characteristics of an effective system', Journal of Advanced Nursing, 21: 551-60.

A. Oakley, J. Popay and P. Watson (1996), Variations in Health: What can the Department of Health and the NHS Do? Social Science Research Unit, Institute of Education, London and Public Health Research and Resource Centre, University of Salford

L. Reynolds (1996), 'A qualitative evaluation of the post-accident notification system to health visitors', Journal of Advanced Nursing, 23: 97-105.

E. Rolls (1992), 'Do the health visitor's professional training and bureaucratic responsibilities separate her from the women she is serving?', Women's Studies International Forum, 15: 397-404.

M. Sheppard (1996), 'Depression in the work of British health visitors: clinical facets', Social Science and Medicine, 43: 1637-48.

P. Stubbs (1993), “Ethnically sensitive" or "anti-racist"? Models for health research and service delivery', in W. I. U. Ahmad (ed.), 'Race' and Health in Contemporary Britain Open University Press, Buckingham.

A. Symonds (1991), 'Angels and interfering busybodies: the social construction of two occupations', Sociology of Health and Illness, 13: 249-64.

N. P. K. Torkington (1995), 'Black migrant women and health', Women's Studies International Forum, 18: 153-8.

J. Welshman (1996), "In search of the "problem family": public health and social work in England and Wales 1940-1970', Social History of Medicine, 9: 447-65.

R. Wiles and J. Robison (1994), 'Teamwork in primary care: the views and experiences of nurses, midwives and health visitors', Journal of Advanced Nursing, 20: 324-30.

C. Willard (1996), 'The nurse's role as patient advocate: obligation or imposition?', Journal of Advanced Nursing, 24: 60-6. 